\title{
STABILITY OF THE POINCARÉ BUNDLE
}

\author{
INDRANIL BISWAS, TOMÁS L. GÓMEZ, AND NORBERT HOFFMANN
}

\begin{abstract}
Let $X$ be an irreducible smooth projective curve, of genus at least two, over an algebraically closed field $k$. Let $\mathcal{M}_{G}^{d}$ denote the moduli stack of principal $G$-bundles over $X$ of fixed topological type $d \in \pi_{1}(G)$, where $G$ is any almost simple affine algebraic group over $k$. We prove that the universal bundle over $X \times \mathcal{M}_{G}^{d}$ is stable with respect to any polarization on $X \times \mathcal{M}_{G}^{d}$. A similar result is proved for the Poincaré adjoint bundle over $X \times M_{G}^{d, \text { rs }}$, where $M_{G}^{d, \text { rs }}$ is the coarse moduli space of regularly stable principal $G$-bundles over $X$ of fixed topological type $d$.
\end{abstract}

\section{INTRODUCTION}

Let $X$ be a compact connected Riemann surface of genus $g_{X} \geq 2$. Fix an integer $r \geq 2$ and a holomorphic line bundle $L$ over $X$ of degree $d$ such that $r$ is coprime to $d$. Let $M_{r, L}$ denote the coarse moduli space of all stable vector bundles $E$ over $X$ with $\operatorname{rank}(E)=r$ and $\bigwedge^{r} E \cong L$. A vector bundle over $X \times M_{r, L}$ is called a Poincaré bundle if its restriction to each closed point $[E] \in M_{r, L}$ is isomorphic to $E$. It is known that Poincaré bundles over $X \times M_{r, L}$ exist, and that any two of them differ by tensoring with a line bundle pulled back from $M_{r, L}$. Balaji, Brambila-Paz and Newstead proved in [BBN] that each Poincaré bundle over $X \times M_{r, L}$ is stable with respect to any polarization on $X \times M_{r, L}$. This result allows to use Poincaré bundles to study moduli spaces of vector bundles on the smooth projective varieties $M_{r, L}$ and $X \times M_{r, L}$, as constructed in [Ma]. It also provides an interesting metric on the Poincaré bundle via the Donaldson-Uhlenbeck-Yau correspondence [Do, UY].

The same question can be asked more generally for moduli spaces of principal $G^{-}$ bundles over $X$. These moduli spaces are no longer smooth projective, and Poincaré $G$-bundles need not exist [BH3]. But over the open locus of regularly stable $G^{-}$ bundles, a Poincaré $G^{\text {ad }}$-bundle always exists, where $G^{\text {ad }}$ denotes the quotient of $G$ modulo its center, and the question whether it is stable still makes sense. For orthogonal and symplectic bundles, this stability is proved in [BG].

Now let $X$ be an irreducible smooth projective curve of genus $g_{X} \geq 2$ over an algebraically closed field $k$. Let $G$ be a smooth connected almost simple algebraic group over $k$. Let $\mathcal{M}_{G}^{d}$ be a connected component of the moduli stack of principal $G^{-}$ bundles over $X$; these connected components are indexed by the elements $d \in \pi_{1}(G)$. From this point of view, we approach the above stability question in this paper. In Section 2, we prove our main result, Theorem 2.2, which states the following:

Theorem. The universal principal $G$-bundle over $X \times \mathcal{M}_{G}^{d}$ is stable with respect to any polarization on $X \times \mathcal{M}_{G}^{d}$.

2000 Mathematics Subject Classification. 14H60, 14D23, 14D20.

Key words and phrases. Moduli stack, Poincaré bundle, stability, moduli space. 
The proof uses the description of $\mathcal{M}_{G}^{d}$ provided by [KNR, BL, BLS], based on the uniformization theorem of Drinfeld and Simpson [DS, Theorem 3]. We first prove as Proposition 2.1 that the restriction of the universal principal $G$-bundle to the slice $\{x\} \times \mathcal{M}_{G}^{d}$ is semistable for any point $x$ on $X$. From this we deduce Theorem 2.2.

Section 3 deals with consequences concerning the coarse moduli scheme $M_{G}^{d}$. In

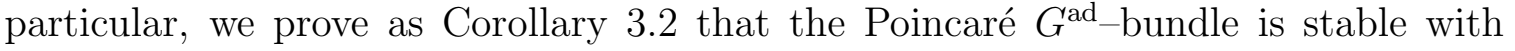
respect to any polarization. Along the way, we again obtain that the restriction of

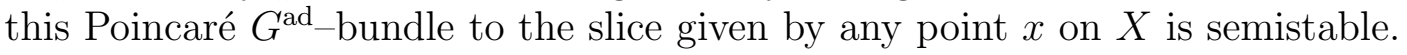

Acknowledgements. The first author is supported by a J. C. Bose Fellowship. The second author acknowledges funding from the Spanish MICINN (grants MTM201679400-P, PID2019-108936GB-C21, and ICMAT Severo Ochoa projects SEV-20150554 and CEX2019-000904-S), the 7th European Union Framework Programme (Marie Curie IRSES grant 612534 project MODULI) and CSIC (2019AEP151 and Ayuda extraordinaria a Centros de Excelencia Severo Ochoa 20205CEX001). The third author was supported by Mary Immaculate College Limerick through the PLOA sabbatical programme. He thanks the Tata Institute of Fundamental Research in Bombay for its hospitality.

\section{StABility OVER THE MOdUli STACK}

Let $X$ be an irreducible smooth projective curve over an algebraically closed field $k$. In this section, we allow the base field $k$ to have arbitrary characteristic.

Let $G$ be a smooth connected reductive algebraic group over $k$. Let $\mathcal{M}_{G}$ denote the moduli stack of principal $G$-bundles over $X$. This stack is smooth over $k$, and its connected components $\mathcal{M}_{G}^{d}$ are indexed by the elements

$$
d \in \pi_{1}(G):=\Lambda_{T_{G}} / \Lambda_{\text {coroots }}
$$

according to [BLS, Proposition 1.3] and [Ho, Theorem 5.8]. Here $\Lambda_{T_{G}}=\operatorname{Hom}\left(\mathbb{G}_{\mathrm{m}}, T_{G}\right)$ is the cocharacter lattice of a maximal torus $T_{G} \subseteq G$, and $\Lambda_{\text {coroots }} \subseteq \Lambda_{T_{G}}$ is the coroot lattice. If $k=\mathbb{C}$, then $\pi_{1}(G)$ coincides with the topological fundamental group.

From now on, we assume that $G$ is almost simple. There is a natural homomorphism

$$
\operatorname{Pic}\left(\mathcal{M}_{G}^{d}\right) \longrightarrow \mathbb{Z}
$$

called central charge, whose kernel and cokernel are both finite. Its definition will be recalled in the proof of Proposition 2.1 below. This central charge has been constructed in [KN, Theorem 2.4] and [BLS, Proposition 1.5] for the case $k=\mathbb{C}$, and in [Fa, Theorem 17] and [BH1, Theorem 5.3.1] for arbitrary characteristic.

Let $\mathcal{L}$ be a line bundle over an open substack $\mathcal{U} \subseteq \mathcal{M}_{G}^{d}$. We say that $\mathcal{U}$ is big if its complement has codimension at least 2 . Then $\mathcal{L}$ extends uniquely to $\mathcal{M}_{G}^{d}$ by $[\mathrm{BH} 3$, Lemma 7.3], and we define $\operatorname{deg} \mathcal{L}$ to be the central charge of this unique extension. For a vector bundle $\mathcal{V}$ of rank $r$ over $\mathcal{U}$, we define $\operatorname{deg} \mathcal{V}$ to be $\operatorname{deg}\left(\bigwedge^{r} \mathcal{V}\right)$.

Let $\mathcal{E} \longrightarrow \mathcal{M}_{G}^{d}$ be a principal $G$-bundle. We say that $\mathcal{E}$ is stable (respectively, semistable) if for every reduction $\mathcal{E} \supset \mathcal{E}_{P} \longrightarrow \mathcal{U}$ to a parabolic subgroup $P \subset G$ over

a big open substack $\mathcal{U} \subseteq \mathcal{M}_{G}^{d}$, and every strictly dominant character $\chi: P \longrightarrow \mathbb{G}_{\mathrm{m}}$ trivial on the center of $G$, the associated line bundle $\mathcal{E}_{P}(\chi)$ over $\mathcal{U}$ satisfies

$$
\left.\operatorname{deg} \mathcal{E}_{P}(\chi)<0 \quad \text { (respectively, } \operatorname{deg} \mathcal{E}_{P}(\chi) \leq 0\right) \text {. }
$$


Note that no choice of a polarization is needed here; it is given by the central charge.

For a principal $G$-bundle over $X \times \mathcal{M}_{G}^{d}$, stability and semistability can be defined similarly, but only after the choice of a polarization. For that, consider homorphisms

$$
\operatorname{Pic}\left(X \times \mathcal{M}_{G}^{d}\right) \longrightarrow \mathbb{Z}
$$

that are locally constant in flat families of line bundles. Since $\operatorname{Pic}\left(\mathcal{M}_{G}^{d}\right)$ is discrete, any such homomorphism is a linear combination of the degree on $X$ and the central charge on $\mathcal{M}_{G}^{d}$; we call it a polarization if it is a positive linear combination. In the above definition of stability, the central charge can be replaced by any such polarization.

Let $\mathcal{E}^{\text {univ }} \longrightarrow X \times \mathcal{M}_{G}$ be the universal principal $G$-bundle, and let $x \in X$ be a closed point. The principal bundle over $\mathcal{M}_{G}$ obtained by restricting $\mathcal{E}^{\text {univ }}$ to the slice $\{x\} \times \mathcal{M}_{G}$, and also its further restriction to $\mathcal{M}_{G}^{d}$, will both be denoted by $\mathcal{E}_{x}^{\text {univ }}$.

Proposition 2.1. Let $G$ be a smooth connected almost simple algebraic group. Let $\mathcal{M}_{G}^{d}$ be a connected component of the moduli stack of principal $G$-bundles over $X$. Then the principal $G$-bundle $\mathcal{E}_{x}^{\text {univ }}$ over $\mathcal{M}_{G}^{d}$ is semistable.

Proof. Choose a closed point $y \in X \backslash\{x\}$, and an isomorphism $\widehat{\mathcal{O}}_{X, y} \cong k[[t]]$. Recall, e.g. from [Fa], that the loop group $L G$ is an ind-scheme over $k$ with

$$
L G(k)=G(k((t))),
$$

the positive loop group is the sub-group scheme $L^{+} G \subseteq L G$ given by

$$
L^{+} G(k)=G(k[[t]]),
$$

and the affine Grassmannian of $G$ is the ind-projective variety

$$
Q_{G}:=L G / L^{+} G \text {. }
$$

The glueing procedure of [KNR, Definition 1.4] defines a morphism

$$
q_{y}: Q_{G} \longrightarrow \mathcal{M}_{G}
$$

that sends the class of $\gamma \in L G$ to the trivial $G$-bundles over the disc $\mathbb{D}:=\operatorname{Spec} \widehat{\mathcal{O}}_{X, y}$ and over $X \backslash\{y\}$, glued by the automorphism $\gamma$ of the trivial $G$-bundle over $\mathbb{D} \backslash\{y\}$. This morphism $q_{y}$ is well-defined because multiplication by $L^{+} G$ from the right can be compensated by changing the trivialization over $\mathbb{D}$. In particular, the pullback of $\mathcal{E}^{\text {univ }}$ from $X \times \mathcal{M}_{G}$ to $X \times Q_{G}$ still comes with a trivialization over $X \backslash\{y\}$. Since $x \neq y$, it follows that the pullback of $\mathcal{E}_{x}^{\text {univ }}$ from $\mathcal{M}_{G}$ to $Q_{G}$ is trivial as well.

Now choose a lift of $d \in \pi_{1}(G)$ to a cocharacter $\delta: \mathbb{G}_{\mathrm{m}} \longrightarrow T_{G} \subseteq G$. We denote by $t^{\delta} \in L G(k)$ the image of the point $t \in L \mathbb{G}_{\mathrm{m}}(k)=k((t))^{*}$ under $\delta$. Let

$$
\pi: \widetilde{G} \longrightarrow G
$$

be the universal cover of $G$. The choice of $\delta$ implies that the composition

$$
Q_{\widetilde{G}} \stackrel{t^{\delta} \cdot \pi(-)}{\longrightarrow} Q_{G} \stackrel{q_{y}}{\longrightarrow} \mathcal{M}_{G}
$$

maps to the component $\mathcal{M}_{G}^{d}$ of $\mathcal{M}_{G}$. This defines a morphism

$$
q_{y}^{\delta}: Q_{\widetilde{G}} \longrightarrow \mathcal{M}_{G}^{d}
$$

which already appears in [BLS, Proposition 1.5]. Due to the uniformization theorem of Drinfeld and Simpson [DS], this morphism $q_{y}^{\delta}$ is surjective. 
Let $\widetilde{\mathfrak{g}}_{\alpha}$ denote the root space in the Lie algebra $\widetilde{\mathfrak{g}}$ of $\widetilde{G}$ corresponding to a root $\alpha$. We denote by $a=1-\alpha$ the affine root of $L \widetilde{G}$ corresponding to the root space

$$
t \cdot \widetilde{\mathfrak{g}}_{-\alpha} \subset L \widetilde{\mathfrak{g}}:=k\left[t, t^{-1}\right] \otimes_{k} \tilde{\mathfrak{g}},
$$

and by $U_{a} \subset L \widetilde{G}$ the corresponding copy of the additive group over $k$. Let

$$
i_{a}: \mathrm{SL}_{2} \longrightarrow L \widetilde{G}
$$

denote the embedding whose image is generated by $U_{a}$ and $U_{-a}$. Then $i_{a}^{-1}\left(L^{+} \widetilde{G}\right)$ is a subgroup of triangular matrices in $\mathrm{SL}_{2}$. Therefore $i_{a}$ induces an embedding

$$
j_{a}: \mathbb{P}^{1} \longrightarrow Q_{\widetilde{G}}
$$

whose image is a simple example of a Schubert variety in $Q_{\widetilde{G}}$. The induced map

$$
\left(j_{a}\right)^{*}: \operatorname{Pic}\left(Q_{\widetilde{G}}\right) \longrightarrow \operatorname{Pic}\left(\mathbb{P}^{1}\right) \cong \mathbb{Z}
$$

is an isomorphism according to [KNR, Proposition 2.3] and [Fa, Corollary 12].

We remark that the composition

$$
\mathbb{P}^{1} \stackrel{j_{a}}{\rightarrow} Q_{\widetilde{G}} \stackrel{q_{y}^{\delta}}{\rightarrow} \mathcal{M}_{G}^{d}
$$

is a generalization to principal $G$-bundles of the Hecke lines in the moduli space of vector bundles introduced in [NR, section 4, p. 397]. For instance, if $G=\mathrm{SL}_{2}$ and $\delta$ is the trivial cocharacter, then this $\mathbb{P}^{1}$-family of principal $\mathrm{SL}_{2}$-bundles over $X$ is the $\mathbb{P}^{1}$-family of all locally free sheaves $E$ of rank 2 over $X$ such that

$$
\mathcal{O}_{X}(-y) \oplus \mathcal{O}_{X} \varsubsetneqq E \varsubsetneqq \mathcal{O}_{X} \oplus \mathcal{O}_{X}(y) \text {. }
$$

The central charge homomorphism in (1) is by definition the composition

$$
\operatorname{Pic}\left(\mathcal{M}_{G}^{d}\right) \stackrel{\left(q_{y}^{\delta}\right)^{*}}{\longrightarrow} \operatorname{Pic}\left(Q_{\widetilde{G}}\right) \stackrel{\left(j_{a}\right)^{*}}{\longrightarrow} \operatorname{Pic}\left(\mathbb{P}^{1}\right) \stackrel{\operatorname{deg}}{\longrightarrow} \mathbb{Z} .
$$

This homomorphism does not depend on the choices made in its construction.

Choosing an embedding $G \hookrightarrow \mathrm{SL}_{N}$, it is easy to see that

$$
H^{0}\left(Q_{\widetilde{G}}, L\right) \neq 0
$$

holds for some $L \in \operatorname{Pic}\left(Q_{\widetilde{G}}\right)$ with $\operatorname{deg}\left(L_{\mid \mathbb{P}^{1}}\right)>0$; indeed, it holds for all such $L$ according to [Fa, Theorem 7]. Since $Q_{\widetilde{G}}$ is an inductive limit of reduced and irreducible projective Schubert varieties, we can conclude that

$$
H^{0}\left(Q_{\widetilde{G}}, L\right)=0
$$

holds for all $L \in \operatorname{Pic}\left(Q_{\widetilde{G}}\right)$ with $\operatorname{deg}\left(L_{\mid \mathbb{P}^{1}}\right)<0$.

Let $\mathcal{E}_{x}^{\text {univ }} \supset \mathcal{E}_{P} \longrightarrow \mathcal{U}$ be a reduction of $\mathcal{E}_{x}^{\text {univ }}$ to a parabolic subgroup $P \subset G$ over a big open substack $\mathcal{U} \subseteq \mathcal{M}_{G}^{d}$. By [Ra, Remark 2.2], it suffices to prove

$$
\operatorname{deg} \operatorname{ad}\left(\mathcal{E}_{P}\right) \leq 0
$$

for the adjoint vector bundle $\operatorname{ad}\left(\mathcal{E}_{P}\right) \longrightarrow \mathcal{U}$. This degree is by definition the central charge of the unique line bundle $\mathcal{L} \longrightarrow \mathcal{M}_{G}^{d}$ that extends the top exterior power

$$
\bigwedge^{\operatorname{dim}(P)} \operatorname{ad}\left(\mathcal{E}_{P}\right) \longrightarrow \mathcal{U}
$$


The inclusion $\operatorname{ad}\left(\mathcal{E}_{P}\right) \subset \operatorname{ad}\left(\mathcal{E}_{x}^{\text {univ }}\right)$ induces an embedding of $\mathcal{L}_{\mid \mathcal{U}}$ into the vector bundle

$$
\mathcal{V}:=\bigwedge^{\operatorname{dim} P} \operatorname{ad}\left(\mathcal{E}_{x}^{\text {univ }}\right) \longrightarrow \mathcal{M}_{G}
$$

This embedding over $\mathcal{U}$ extends to a generically injective morphism from $\mathcal{L}$ to $\mathcal{V}$ over $\mathcal{M}_{G}^{d}$ due to Hartogs' theorem. By pullback, we obtain a nonzero morphism from

$$
L:=\left(q_{y}^{\delta}\right)^{*} \mathcal{L} \longrightarrow Q_{\widetilde{G}}
$$

to the vector bundle $\left(q_{y}^{\delta}\right)^{*} \mathcal{V} \longrightarrow Q_{\widetilde{G}}$. However, this vector bundle is trivial, since the principal $G$-bundle $\left(q_{y}\right)^{*} \mathcal{E}_{x}^{\text {univ }} \longrightarrow Q_{G}$ is trivial. Therefore,

$$
H^{0}\left(Q_{\widetilde{G}}, L^{\text {dual }}\right) \neq 0,
$$

and hence $\operatorname{deg}\left(L_{\mid \mathbb{P}^{1}}\right) \leq 0$. But $\operatorname{deg}\left(L_{\mid \mathbb{P}^{1}}\right)$ is by definition the central charge of $\mathcal{L}$.

Theorem 2.2. If the curve $X$ has genus $g_{X} \geq 2$, then the universal bundle $\mathcal{E}^{\text {univ }}$ over $X \times \mathcal{M}_{G}^{d}$ is stable with respect to any polarization on $X \times \mathcal{M}_{G}^{d}$.

Proof. The restriction of $\mathcal{E}^{\text {univ }}$ to any point in $X$ is semistable by Proposition 2.1. The restriction of $\mathcal{E}^{\text {univ }}$ to a general point in $\mathcal{M}_{G}^{d}$ is stable, because the open locus $\mathcal{M}_{G}^{d} \supset \mathcal{M}_{G}^{d, \mathrm{~s}}$ of stable $G$-bundles over $X$ is nonempty for $g_{X} \geq 2$. These two facts imply the theorem, as the proof of [BBN, Lemma 2.2] shows.

Remark 2.3. If $G$ is only semisimple, then its universal cover $\widetilde{G}$ is a product of $s \geq 2$ almost simple factors. Each of them defines a central charge, and their direct sum

$$
\operatorname{Pic}\left(\mathcal{M}_{G}^{d}\right) \longrightarrow \mathbb{Z}^{s}
$$

again has finite kernel and cokernel. Proposition 2.1 remains true in this generality, now with respect to any polarization, because $H^{0}\left(Q_{\widetilde{G}}, L\right)=0$ for every line bundle $L$ over $Q_{\widetilde{G}}$ with at least one negative central charge. Consequently, Theorem 2.2, and the two corollaries in the next section, all generalize to the semisimple case as well.

\section{Stability over the COARSE MOduli SPACE}

In this section, we assume that the base field is $k=\mathbb{C}$. Let $M_{G}$ denote the coarse moduli space of semistable principal $G$-bundles over $X$. Its connected components $M_{G}^{d}$ are irreducible normal projective varieties, indexed by $d \in \pi_{1}(G)$.

A principal $G$-bundle $E$ over $X$ is called regularly stable if $E$ is stable and $\operatorname{Aut}(E)=$ $Z_{G}$, the center of $G$. We denote by $M_{G}^{d, \text { rs }} \subset M_{G}^{d}$ the open locus of regularly stable principal $G$-bundles. The quotient $G / Z_{G}$ will be denoted by $G^{\text {ad }}$. The appropriate

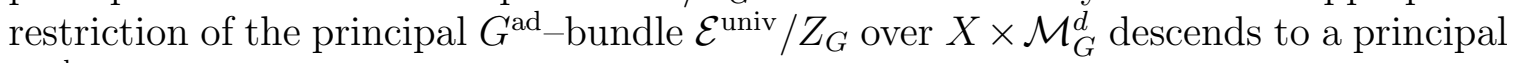
$G^{\text {ad_bundle }}$

$$
\mathcal{E}^{\text {Poinc }} \longrightarrow X \times M_{G}^{d, \text { rs }}
$$

Let $\mathcal{E}_{x}^{\text {Poinc }}$ denote the restriction of $\mathcal{E}^{\text {Poinc }}$ to $\{x\} \times M_{G}^{d, \text { rs }}$ for a closed point $x \in X$.

From now on, we again assume that $G$ is almost simple. We also assume that the curve $X$ has genus $g_{X} \geq 2$, and even $g_{X} \geq 3$ if $G^{\text {ad }} \cong \mathrm{PGL}_{2}$. Then $M_{G}^{d, \text { rs }}$ is the smooth locus of $M_{G}^{d}$ by [BH2, Corollary 3.4]. In particular, the complement $M_{G}^{d} \backslash M_{G}^{d, \text { rs }}$ has 
codimension at least two. Therefore, $\operatorname{Pic}\left(M_{G}^{d, \text { rs }}\right)$ embeds as a subgroup into $\operatorname{Pic}\left(\mathcal{M}_{G}^{d}\right)$ (cf. [BLS, $\S(13.1)]$ ), and the central charge restricts to a natural homomorphism

$$
\operatorname{Pic}\left(M_{G}^{d, \mathrm{rs}}\right) \longrightarrow \mathbb{Z}
$$

whose kernel and cokernel are still both finite. This again provides a polarization on $M_{G}^{d, \text { rs }}$, so there is no need to choose one in dealing with stability over $M_{G}^{d, \mathrm{rs}}$.

As direct consequences of Proposition 2.1 and Theorem 2.2, we obtain the following.

Corollary 3.1. The principal $G^{\text {ad }}$-bundle $\mathcal{E}_{x}^{\text {Poinc }}$ over $M_{G}^{d, \text { rs }}$ is semistable.

Corollary 3.2. The Poincaré adjoint bundle $\mathcal{E}^{\text {Poinc }}$ over $X \times M_{G}^{d, \mathrm{rs}}$ is stable with respect to any polarization on $X \times M_{G}^{d, \mathrm{rs}}$.

Remark 3.3. If the base field $k=\bar{k}$ has characteristic $p>0$, then Corollary 3.1 and Corollary 3.2 remain valid at least for $g_{X} \geq 4$, because the locus of regularly stable principal $G$-bundles $E$ over $X$ is still open by [BH3, Proposition 2.3], and its complement still has codimension at least two by [BH3, Theorem 2.5].

\section{REFERENCES}

[BBN] V. Balaji, L. Brambila-Paz and P. E. Newstead, Stability of the Poincaré bundle, Math. Nachr. 188 (1997), 5-15.

[BG] I. Biswas and T. L. Gómez, Stability of symplectic and orthogonal Poincaré bundles, J. Geometry Phys. 76 (2014), 97-106.

[BL] A. Beauville and Y. Laszlo, Conformal blocks and generalized theta functions, Comm. Math. Phys. 164 (1994), 385-419.

[BLS] A. Beauville, Y. Laszlo and C. Sorger, The Picard group of the moduli of $G$-bundles on a curve, Compositio Math. 112 (1998), 183-216.

[BH1] I. Biswas and N. Hoffmann, The line bundles on moduli stacks of principal bundles on a curve, Doc. Math. 15 (2010), 35-72.

[BH2] I. Biswas and N. Hoffmann, A Torelli theorem for moduli spaces of principal bundles over a curve, Ann. Inst. Fourier 62 (2012), 87-106.

[BH3] I. Biswas and N. Hoffmann, Poincaré families of $G$-bundles on a curve, Math. Ann. 352 (2012), 133-154

[Do] S. K. Donaldson, Infinite determinants, stable bundles and curvature, Duke Math. J. 54 (1987), 231-247.

[DS] V. G. Drinfeld and C. Simpson, B-structures on $G$-bundles and local triviality, Math. Res. Lett. 2 (1995), 823-829.

[Fa] G. Faltings, Algebraic loop groups and moduli spaces of bundles, Jour. Eur. Math. Soc. 5 (2003), 41-68.

[Ho] N. Hoffmann, On moduli stacks of G-bundles over a curve, Affine flag manifolds and principal bundles, 155-163, Trends Math., Birkhäuser/Springer Basel AG, Basel, 2010.

[KN] S. Kumar and M. S. Narasimhan, Picard group of the moduli spaces of G-bundles, Math. Ann. 308 (1997), 155-173.

[KNR] S. Kumar, M. S. Narasimhan and A. Ramanathan, Infinite Grassmannians and moduli spaces of $G$-bundles, Math. Ann. 300 (1994), 41-75.

[Ma] M. Maruyama, Moduli of stable sheaves I, J. Math. Kyoto Univ. 17 (1977), 91-126.

[NR] M.S. Narasimhan and S. Ramanan, Deformations of the moduli of vector bundles, Ann. Math. 101 (1975), 391-417.

[Ra] A. Ramanathan, Stable principal bundles on a compact Riemann surface, Math. Ann. 213 (1975), 129-152. 
[UY] K. Uhlenbeck and S.-T. Yau, On the existence of Hermitian-Yang-Mills connections in stable vector bundles. Comm. Pure Appl. Math. 39 (1986), suppl., S257-S293.

School of Mathematics, Tata Institute of Fundamental Research, Homi Bhabha ROAD, BOMBAY 400005, India

Email address: indranil@math.tifr.res.in

Instituto de Ciencias Matemáticas (CSIC-UAM-UC3M-UCM), Nicolás Cabrera 15, Campus Cantoblanco UAM, 28049 Madrid, Spain

Email address: tomas.gomez@icmat.es

Department of Mathematics and Computer Studies, Mary Immaculate College, South Circular Road, Limerick, Ireland

Email address: norbert.hoffmann@mic.ul.ie 